\title{
Reliable Nanofabrication of Single-Crystal Diamond Photonic Nanostructures for Nanoscale Sensing
}

\author{
Mariusz Radtke ${ }^{+}(\mathbb{D})$, Richard Nelz (1), Abdallah Slablab (i) and Elke Neu *(i) \\ Faculty of Natural Sciences and Technology, Saarland University, Physics, Campus E2.6, \\ 66123 Saarbrücken, Germany \\ * Correspondence: elkeneu@physik.uni-saarland.de \\ + Current address: Department of Organic and Macromolecular Chemistry, Ghent University Krijgslaan 281, \\ building S4, 9000 Gent, Belgium.
}

Received: 26 September 2019; Accepted: 17 October 2019; Published: 24 October 2019

\begin{abstract}
In this manuscript, we outline a reliable procedure to manufacture photonic nanostructures from single-crystal diamond (SCD). Photonic nanostructures, in our case SCD nanopillars on thin $(<1 \mu \mathrm{m})$ platforms, are highly relevant for nanoscale sensing. The presented top-down procedure includes electron beam lithography (EBL) as well as reactive ion etching (RIE). Our method introduces a novel type of inter-layer, namely silicon, that significantly enhances the adhesion of hydrogen silsesquioxane (HSQ) electron beam resist to SCD and avoids sample charging during EBL. In contrast to previously used adhesion layers, our silicon layer can be removed using a highly-selective RIE step, which is not damaging HSQ mask structures. We thus refine published nanofabrication processes to ease a higher process reliability especially in the light of the advancing commercialization of SCD sensor devices.
\end{abstract}

Keywords: top-down nanofabrication; single-crystal diamond; HSQ; electron beam lithography; inductively coupled-reactive ion etching (ICP-RIE)

\section{Introduction}

In recent decades, the use of optically active point defect, i.e., color centers, in single-crystal diamond (SCD) as atom-sized, solid-based quantum systems has emerged in various fields [1,2]. Applications span from quantum metrology (temperature [3], strain [4], electric [5] and magnetic fields [6]) to using color centers as spin qubits in quantum computing [7] and single photon sources for quantum communication [8,9]. The outstanding color center in diamond is the $\mathrm{NV}^{-}$center due to its optically readable spin [10] and usage as sensor. For many of these applications, color centers will be incorporated into photonic nanostructures e.g., nanopillars [9] to ease fluorescence detection from the color centers and to enable, e.g., scanning a color center close to a sample surface [6].

SCD's wide indirect bandgap of $\sim 5.45 \mathrm{eV}$ makes undoped SCD a good insulator [11]. Moreover, SCD shows a high chemical inertness. Both properties render fabricating SCD nanostructures challenging: Top-down methods for nanofabrication will use lithography, typically electron beam lithography (EBL), as well as etching. As the high chemical inertness of SCD prevents wet etching, only plasma etching, typically inductively coupled reactive ion etching (ICP-RIE), is applicable. Moreover, the insulating nature of SCD renders EBL highly challenging due to uncontrolled sample charging and the resulting deflection of the electron beam. A peculiarity of SCD nanofabrication arises also from the fact that only certain materials can efficiently serve as an etch mask in the high-bias, high-density plasmas necessary for anisotropic SCD etching [12]. The now state-of-the-art masks for SCD nanostructuring are EBL written structures consisting of hydrogen silsesquioxane (HSQ). HSQ is stable in anisotropic etch plasmas used for SCD etching; it etches an order of 
magnitude slower than SCD using typical etching recipes [12]. In general, HSQ enables creating very small mask structures down to $20 \mathrm{~nm}$ [13]. SCD structures etched using HSQ masks show smooth sidewalls [12]. Smooth sidewalls ensure low light scattering from photonic structures and defined waveguide properties. Consequently, HSQ masks enable etching almost cylindrical pillars with optimized shape and well-defined photonic properties $[6,9,12,14,15]$. On the other hand, we find that HSQ has a non-optimal adhesion to SCD. In previous work, this challenge has often been addressed using metallic inter-layers between HSQ and SCD, e.g., titanium [16]. However, even very thin $(<1 \mathrm{~nm})$ metallic residuals on SCD surfaces strongly disturb color centers placed shallowly below the surface [17]. Consequently, any metallic residues are detrimental for the process and a metal-free process is highly desirable. Often, removing the metallic layer also requires wet chemical removal [16] or the use of toxic etch gases such as chlorine [18]. The first can leave trace amounts of the etchant on the SCD surface and the second is technically demanding considering safety and reactor corrosion.

In this manuscript, we present a method to overcome two previously not satisfactorily addressed challenges in SCD nanofabrication, namely sample charging as well as non-optimal resist adhesion. We used the optimized process to fabricate SCD scanning probes, namely nanopillars on thin holding platforms [6,16]. We thus reliably fabricated SCD nanostructures easing, e.g., commercial fabrication of SCD scanning probes. Figure 1 depicts the steps of our nanofabrication process. We start with a clean SCD sample with a shallow $\mathrm{NV}^{-}$layer (details on sample pre-treatment see Section 2) as mandatory for high resolution sensing. We use electron beam evaporation of silicon on SCD to form a de-charging and adhesive layer (Figure $1 b$, Section 3). This layer will enable highly reliable spin coating of HSQ (Figure 1c) as well as EBL (Figure 1d, Section 3). Subsequently, we remove the silicon adhesion layer selectively using ICP-RIE without damage to the HSQ mask (Figure 1e) and perform ICP-RIE of SCD to form the desired structures (Figure 1f, Section 4). Our method eases manufacturing complex structures, e.g., in our case, nanopillars (diameter $200 \mathrm{~nm}$ ), on top of SCD platforms (size of the platform $\sim 3 \times 20 \mu \mathrm{m}$ ): In the first structuring step, we form the platforms (Figure 1f). The silicon adhesion layer survives the subsequent wet-chemical removal of the HSQ mask (Figure 1g) and can be reused for a second round of processing (Figure 1h-1). In this second processing, we form the pillars. We note that, when etching the platforms, the HSQ mask protects NV centers in the whole area of the micrometer-sized platform. During pillar etching, only NVs protected by the pillar mask survive the process and will be used as nanoscale sensors. The method presented here has been filed for a patent (EP19198772.6). 
Schematics of the fabrication process

(a)

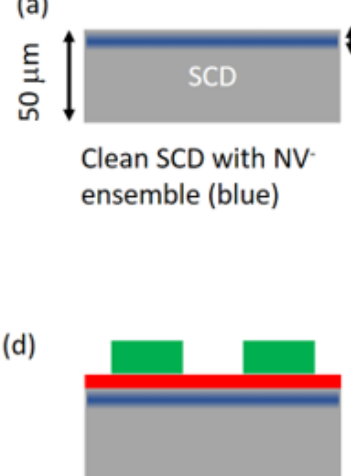

EBL and mask development

(g)

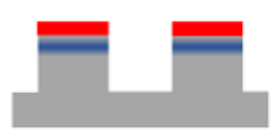

Mask removal

(j)

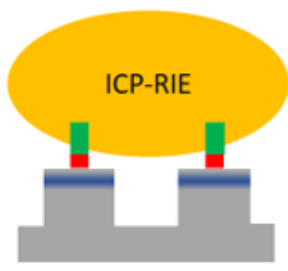

$\mathrm{SF}_{6}$ pulse (b)

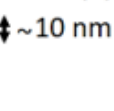

\section{Deposition of}

adhesion layer (red)

(e)

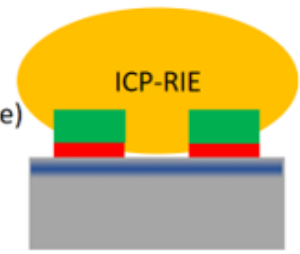

$\mathrm{SF}_{6}$ pulse

(h)

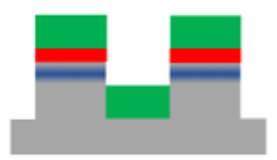

Spin coat new resist

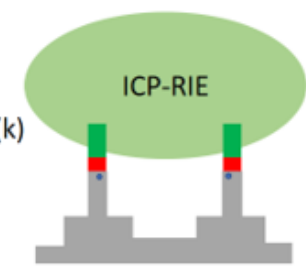

Diamond Etch (c)

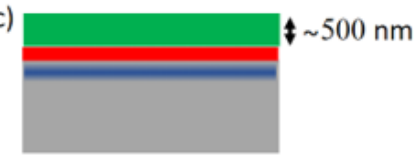

Spin coating of resist (green)

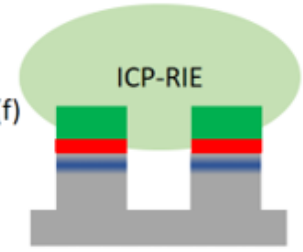

Diamond etch

(i)

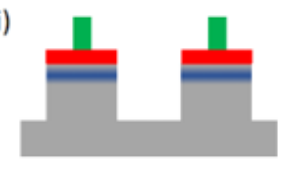

EBL and development

(I)

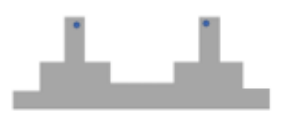

Clean Diamond Sensor devices

Figure 1. (a) The process starts with an SCD sample with a shallow layer of NV centers. Subsequently, we deposit (b) a silicon adhesion layer and (c) spin coat HSQ. (d) We use electron beam lithography (EBL) to structure the HSQ resist and obtain masks for the platforms. Using ICP-RIE, (e) we remove the silicon layer in-between the HSQ mask and (f) perform the structuring etch for SCD. (g) We finalize platform structuring via removing residual HSQ. We now (h) spin coat HSQ onto the remaining silicon layer and (i) perform EBL again. We repeat the ICP-RIE procedure, firstly (j) removing the silicon layer and then (k) etching the pillars into SCD. In a last step, (1) we remove residual HSQ as well as silicon to obtain clean SCD devices.

\section{Sample Pre-Treatment}

We purchased high-purity, (100)-oriented, chemical vapor deposited, SCD from Element Six (electronic grade quality, $[\mathrm{N}]^{s}<5 \mathrm{ppb}, \mathrm{B}<1 \mathrm{ppb}$ ). As we aimed for free standing SCD devices consisting of nanopillars on platforms, the SCD plates (size $2 \times 4 \mathrm{~mm}^{2}$ ) were polished down to thickness of $50 \mu \mathrm{m}$ (Delaware Diamond Knives, Wilmington, DE, US). The SCD surface showed an initial roughness of $\mathrm{R}_{a}<3 \mathrm{~nm}$. As the mechanical polishing of the SCD can leave highly contaminated surfaces, we first wiped the sample surface using clean-room wipes and performed cleaning in an ultrasonic bath (solvents: isopropanol and acetone). We then cleaned the sample in boiling acids (1:1:1 mixture of sulfuric acid, perchloric acid and nitric acid, $5 \mathrm{~mL}$ each).

Mechanical polishing is suspected to introduce damage that potentially extends several micrometers deep into the SCD material $[19,20]$. To remove this potentially damaged and strained material, we applied ICP-RIE to our SCD samples. We avoided the use of toxic or corrosive gases in the process following our previously published routine [21]. We used a Plasmalab 100 ICP-RIE reactor (Oxford instruments, Abington, UK) and removed the topmost 3-5 $\mu \mathrm{m}$ of SCD from each side. We 
used a combination of $\mathrm{SF}_{6}, \mathrm{O}_{2}$, Ar biased plasmas with mixed RF and ICP discharges. Following recent approaches [22,23], we terminated the etching using low-damage, $0 \mathrm{~V}$ bias plasma with pure oxygen. The use of such soft etching was motivated by the potential close-to-surface damage due to highly biased ICP etching [24]. We typically obtain very smooth surfaces with an rms roughness of $\sim 1 \mathrm{~nm}$.

Using the above described procedure, we avoided creating NV centers in potentially damaged SCD. We formed a homogeneous layer of $\mathrm{NV}^{-}$centers by implanting nitrogen ions with a density of $2 \times 10^{11}$ ions $/ \mathrm{cm}^{2}$ and an energy of $6 \mathrm{keV}$. During the implantation, the sample was tilted by $7^{\circ}$ with respect to the ion beam to avoid ion channeling. The SCD sample was then annealed in vacuum at $800^{\circ} \mathrm{C}$ followed by an acid clean. This treatment will typically leave our sample with a mixed oxygen termination on the surface [25]. We found a contact angle for water of $67^{\circ}$ [26], indicating a hydrophilic surface. As the electron beam resist we want to apply to the SCD was dissolved in methyl isobutyl ketone, a polar molecule, the resist's solvent had high affinity to hydrophilic surfaces. Despite the, in principle, fitting surface termination of the SCD sample, we observed non-reliable adhesion when applying HSQ to the SCD surface.

\section{Deposition of Adhesion Layer and HSQ Mask Structuring}

Motivated by the lack of reliable adhesion of HSQ to clean SCD surfaces, we explored silicon as an inter-layer. We expect this layer to foster adhesion between polysilicate HSQ resist and the native oxide $\left(\mathrm{SiO}_{2}\right)$ on the layer. To deposit the silicon adhesion layer, we used electron beam evaporation at a pressure of $10^{-6}$ Torr and $10 \mathrm{kV}$ acceleration voltage with elliptical beam scanning mode in an electron beam evaporator "Pfeiffer Classic 500 L" machine. For the present work, we chose a thickness of the silicon layer of $25 \mathrm{~nm}$. We note that we also found sputtered silicon layers to efficiently foster adhesion between SCD and HSQ. However, the SCD surface was attacked during the sputtering process. This in our case led to excess blinking and bleaching of $\mathrm{NV}^{-}$centers in the final sensing devices and rules out this approach for our application. We also note that we tested spin coating Ti-prime as an adhesion promoter but did not obtain reliable results. We furthermore tested chromium layers as alternative to quickly oxidizing titanium layers [16]. Using this approach, we faced micromasking effects most probably arising from the incomplete, non-reliable wet-chemical removal of chromium layers. We note that our silicon layers still enable efficient HSQ adhesion weeks after deposition and storage under ambient conditions. We consequently conclude that the formation of a native oxide layer on the silicon, which will occur during storage at ambient conditions, is not detrimental. Thus, technically speaking, evaporation of silicon layers can be performed in batch processes for several SCD samples, which eases the fabrication workflow and reduces machine time. The evaporated silicon layers are very uniform and show a low roughness, as evidenced by AFM. We note that adhesion of the silicon layer to SCD was very reliable and we never observed any hints of cracking or peeling throughout the whole process, deduced from AFM and SEM microscopy. We have processed more than 10 samples using the here described method and no SEM images showed peeling or cracking of the silicon layer. To avoid any damage of the surface caused by contact with the tip (AFM) or amorphous carbon deposition (from SEM chamber), no routine checks were performed prior each fabrication step. We note we also observed the surface topography of samples covered with HSQ layers. In case of any silicon peeling, this would be evident by folding of spin-coated HSQ.

To manufacture etch masks based on HSQ, we used Fox 16 resist (Dow Corning, Midland, MI, USA), which we spin-coated onto the SCD plate. To ease handling of our small SCD plates, we glued them to silicon carrier chips using crystalbond adhesive. We note that the silicon carrier can be removed at the end of our nanofabrication process using acetone to dissolve crystalbond without damage to the SCD nanostructures. Prior to spin coating, we heated the SCD sample on the silicon carrier for $10 \mathrm{~min}$ at $120^{\circ} \mathrm{C}$ to remove any moisture from the surface. We applied roughly $0.3 \mathrm{~mL}$ of Fox 16 solution to the SCD plate and spin-coated it at $1000 \mathrm{rpm}$ for $10 \mathrm{~s}$ then increasing rotation speed to $3300 \mathrm{rpm}$ for $60 \mathrm{~s}$. Subsequently, we pre-baked the sample at $90^{\circ} \mathrm{C}$ for $5 \mathrm{~min}$. We note that great care has been taken to not exceed the shelf life of the Fox 16 resist. As a result of the small size of our samples as 
well as the spin coating on already etched structures in the latter stages of our fabrication process, we can only estimate the thickness of the HSQ, which shows a significant variation from sample to sample. From SEM images of pillar masks on platforms, we estimate a HSQ layer thickness of $\sim 0.9 \mu \mathrm{m}$. Consequently, considering a pillar diameter of $\sim 200 \mathrm{~nm}$, we demonstrate reliable adhesion of HSQ masks with an aspect ratio of 4.5 .

We inserted the SCD plate including the silicon carrier chip into our EBL machine (cold-cathode SEM, Hitachi S45000, Chiyoda, Japan, equipped with RAITH Elphy software). We note that EBL of the spin-coated HSQ layer has to be done directly after spin coating to avoid any reaction of HSQ with air. We performed EBL at $30 \mathrm{kV}$ acceleration voltage and $20 \mu \mathrm{A}$ extracting current. The working distance is kept at $15.3 \mathrm{~mm}$ for $400 \times 400 \mu^{2}$ fields. During our device fabrication, larger structures, namely the rectangular holding platforms (size $\sim 3 \times 20 \mu \mathrm{m}^{2}$ ) as well as masks for nanopillars (diameter $200 \mathrm{~nm}$ ), were of interest. We wrote platforms using longitudinal writing mode and pillars using concentric writing modes. The doses for large structures were established to be optimal as $0.49 \mathrm{mC} / \mathrm{cm}^{2}$ and for pillar structures, with a variation with thickness of the HSQ layer, between 2.24 (planar SCD) and $7 \mathrm{mC} / \mathrm{cm}^{2}$ (pre-structured SCD with, e.g., platforms).

We developed the HSQ in 25\% TMAH solution without swirling the solution. After $20 \mathrm{~s}$, the SCD sample was placed in ultra-pure $18 \mathrm{MOhm} \mathrm{cm}$ MiliQ water and subsequently immersed several times in acetone and isopropanol. We note that the development has to take place directly after removing the SCD plate from the EBL vacuum chamber.

\section{Selective ICP-RIE of Adhesion Layer and SCD Structuring}

A dedicated ICP/RIE plasma sequence based on $\mathrm{O}_{2}$-based etching of SCD, preceded by a short pulse of $\mathrm{SF}_{6}$ plasma, was designed. This sequence first selectively removes the silicon layer between the HSQ-based mask structures and subsequently enables highly anisotropic etching of SCD. The parameters of the plasmas are summarized in Table 1. In the final process, we ran the above mentioned sequence without removing the sample from the ICP-RIE reactor in-between the plasma steps to avoid any contamination.

Reliably removing the silicon layer without any residuals is vital to our process: We observed a partial etch stop as well as strong micromasking when applying the $\mathrm{O}_{2}$-based plasma without applying the $\mathrm{SF}_{6}$ pulse (see Figure $2 \mathrm{a}$ ). We deduced a complete and reliable removal of the silicon adhesion layer from two facts: First, in SEM images taken directly after the $\mathrm{SF}_{6}$ pulse (see Figure $2 \mathrm{~b}$ ), a clear contrast between etched and non-etched areas is visible. We also investigated the $\mathrm{SF}_{6}$-based etching process by means of optical emission spectroscopy shown in Figure 2c. In the spectrum a series of emission lines corresponding to fluoride $\left(\mathrm{F}^{-}\right)$was observed [27]. We attributed the etching of silicon to this $\mathrm{F}^{-}$ions. Second, knowing that the $\mathrm{O}_{2}$ plasma used to etch SCD was not etching the silicon layer, the absence of micromasking and very smooth surfaces in-between the etched structures (see Figure 3a) proved the complete removal of the silicon layer. We note that using pure $\mathrm{SF}_{6}$ is vital to arrive at this result, as introduction of other gases (Argon or Oxygen) at this stage generated severe micromasking. Our $\mathrm{SF}_{6}$ plasma removes the silicon layer while maintaining a 1:20 selectivity in favor of the HSQ mask. For our process, this means that, during removal of the $25 \mathrm{~nm}$ thick silicon adhesion layer, less than $2 \mathrm{~nm}$ of the HSQ mask, which in our case is several hundreds of nanometer thick, will be lost. This result corresponds well to similar plasmas obtained in different systems showing highly selective silicon etching while conserving $\mathrm{SiO}_{2}$ (in our case HSQ) [28]. We furthermore observed no or minor etching of SCD during the $\mathrm{SF}_{6}$ pulse and no roughening of the exposed SCD surface. We confirmed full etching of the silicon layer using Raman spectroscopy and XPS, whereas the latter only showed C1s and O1s peaks. Using EDX, we checked that there is no silicon contamination on the etched SCD after the $\mathrm{O}_{2}$-based plasma (see Figure 4). 
Table 1. Etching plasma parameters. $\mathrm{SF}_{6}$ pulse used to selectively remove the evaporated silicon layer ( $5 \mathrm{~s}$ including plasma ignition step). The $\mathrm{O}_{2}$ plasma is subsequently used as an anisotropic etch for SCD to form the platforms as well as the pillars (10-15 min etching time).

\begin{tabular}{cccccc}
\hline Plasma & $\begin{array}{c}\text { ICP Power } \\
\text { W }\end{array}$ & $\begin{array}{c}\text { RF Power } \\
\text { W }\end{array}$ & $\begin{array}{c}\text { Gas Flux } \\
\text { sccm }\end{array}$ & $\begin{array}{c}\text { Etch Rate } \\
\text { nm/min }\end{array}$ & $\begin{array}{c}\text { Pressure } \\
\text { Pa }\end{array}$ \\
\hline $\mathrm{SF}_{6}$ pulse & 300 & 100 & $\mathrm{SF}_{6}: 25$ & Si: $1072 \mathrm{HSQ}: 52$ & 1.3 \\
$\mathrm{O}_{2}$ plasma & 500 & 200 & $\mathrm{O}_{2}: 50$ & 104 & 1.5 \\
\hline
\end{tabular}
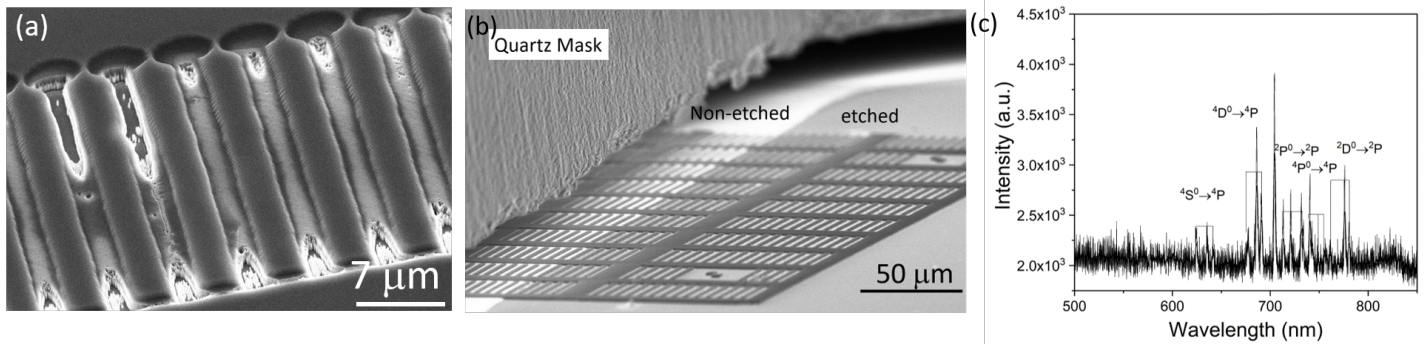

Figure 2. (a) Pattern of holding platforms etched with $\mathrm{O}_{2}$ plasma without applying the $\mathrm{SF}_{6}$ pulse plasma to remove the silicon layer, the SEM image shows the bare SCD structures. Strong micro-masking and corresponding roughening as well as a partial etch stop are visible (b) Scanning electron microscope (SEM) image of SCD surface with HSQ structures, here platforms, after $\mathrm{SF}_{6}$ pulse. A part of the platforms is covered with a quartz plate (marked in the image) during the $\mathrm{SF}_{6}$ pulse. The strongly reduced brightness of the etched surface in contrast to non-etched surface indicates the complete removal of the silicon adhesion layer. We furthermore observe no or minor etching of SCD during the $\mathrm{SF}_{6}$ pulse and no roughening of the exposed SCD surface. (c) Optical emission spectrum of the $\mathrm{SF}_{6}$ pulse plasma step indicating presence of fluoride $\left(\mathrm{F}^{-}\right)$species responsible for selective removal of silicon from the SCD surface.
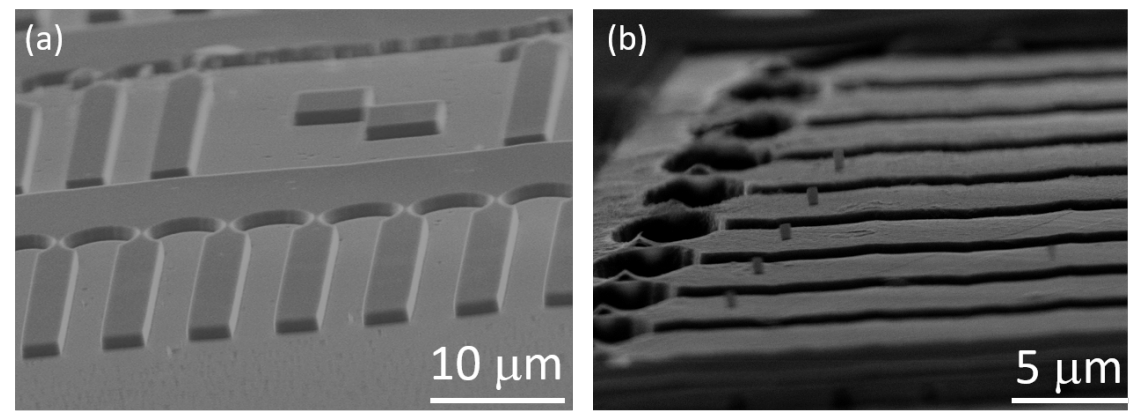

Figure 3. (a) Devices at intermediate stage of the process with etched-in platforms. Note that the SEM image shows the bare SCD structures that have been obtained via removing the adhesion layer using the $\mathrm{SF}_{6}$ pulse followed by anisotropic $\mathrm{O}_{2}$ RIE and subsequent cleaning. (b) HSQ masks for pillars etching written by EBL. The masks are residing on SCD platforms coated by a freshly evaporated silicon layer. Note that the silicon layer is not discernible in the SEM images.

After successfully structuring our SCD platforms, we removed HSQ residuals using HF-based buffered oxide etch by immersion of SCD for $20 \mathrm{~min}$ in the solution. Although this step removed the native oxide from our silicon adhesion layer, the layer itself survived the process, as clearly discernible from the EDX imaging in Figure 4b. Consequently, it can be re-used for consecutive steps. We then spin-coated HSQ again, which in our case formed a layer on top as well as in-between the platforms. We then re-emploedy EBL to create pillar masks on the platforms (see Figure 3b). We repeated the etching to transfer the pillar mask into the SCD platform creating almost cylindrical pillars. 

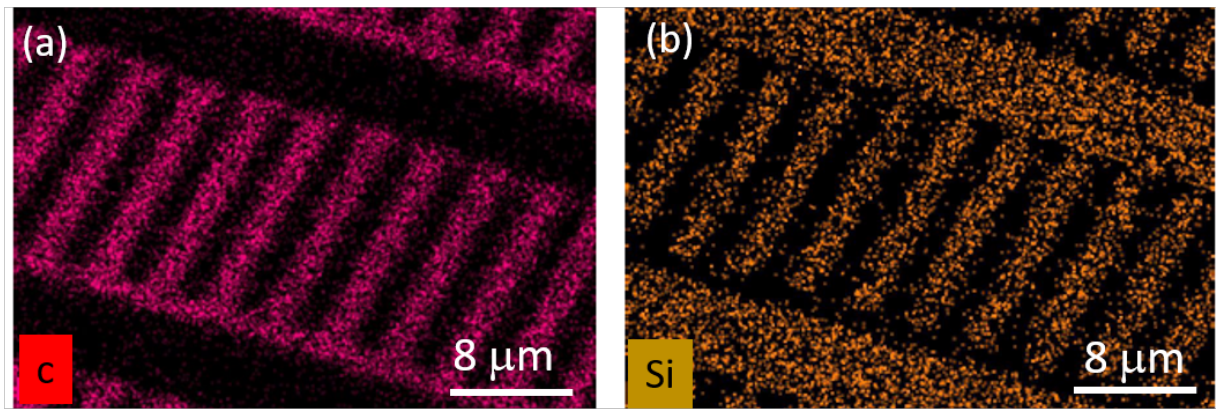

Figure 4. Energy dispersive X-ray (EDX) spectroscopy mapping of SCD cantilevers prepared by the presented method. The EDX mapping has been performed after the $\mathrm{SF}_{6}$ plasma, the $\mathrm{O}_{2}$ plasma etching the SCD structures (parameters see Table 1) and the wet chemical removal of residual HSQ: (a) carbon signal; and (b) silicon signal. The two maps show complementary images, clearly indicating that, in-between the platforms, we find bare SCD (carbon) with no silicon signal while on the platform, the silicon adhesion layer survived and is ready to be used in the next processing step.

\section{Final Devices and Device Characterization}

To obtain clean SCD devices, we removed all HSQ residuals using HF-based buffered oxide etch. We immersed the SCD into buffered oxide etch for $20 \mathrm{~min}$ which removes the HSQ as well as any native oxide on the silicon layer. Afterwards, we immersed the SCD sample in 3M potassium hydroxide at $80{ }^{\circ} \mathrm{C}$ for $30 \mathrm{~min}$ to remove the silicon adhesion layer and revealed the clean SCD structures. After this process, we repeated the three-acid cleaning described above before characterizing the photoluminescence (PL) of $\mathrm{NV}^{-}$centers in the SCD nanostructures. Figure 5a displays devices obtained using this process. We note that, to obtain free standing devices, which we mounted to quartz capillaries as holders (see Figure $5 \mathrm{~b}$ ), the SCD plate has to be thinned from the non-structured side until the devices are fully released. For more details on the mounting, see Reference [16]. To this end, we employed previously published deep-etching routines [21] which are beyond the scope of this manuscript.
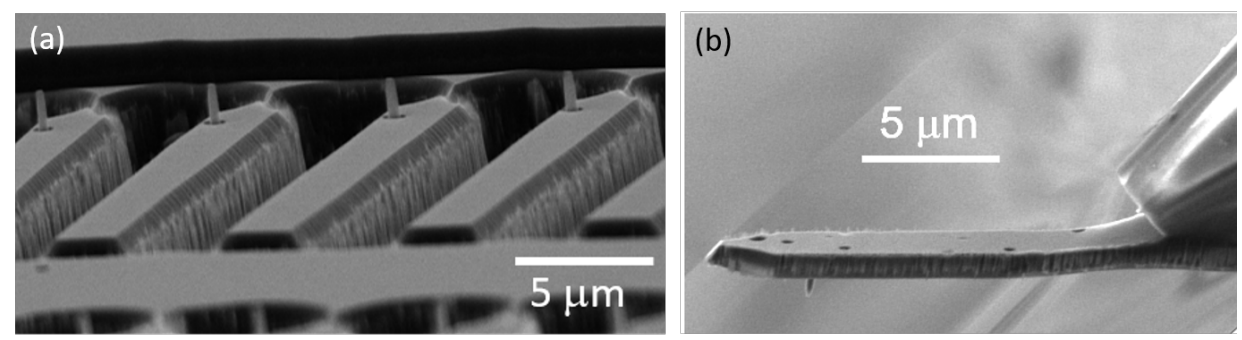

Figure 5. Scanning probe devices manufactured using the described nanofabrication process. (a) Pillars on platforms. The shown devices still need thinning of the SCD plate from the backside to release the devices. (b) Mounted SCD scanning probe. On the right hand side, a quartz capillary is visible that serves as a holder to mount the device to a scanning probe microscope.

To investigate the photonic properties of our SCD nanostructures, we used a custom-built confocal microscope (numerical aperture 0.8). Details of the setup are given in References [22,29-31].

We first measured confocal PL maps of the structures (see Figure $6 \mathrm{a}, \mathrm{b}$ ) excited at $532 \mathrm{~nm}$ with a power of $500 \mu \mathrm{W}$. We clearly observed intense PL ( $\sim 100 \mathrm{kcps})$ originating from single $\mathrm{NV}^{-}$centers in the pillars (see Figure 6a). We estimated the maximum achievable PL of the $\mathrm{NV}^{-}$centers to be $>300$ kcps comparable to previous work [16]. In addition, we investigated the background PL from the etched surface ( $\sim 1 \mathrm{kcps}$ ), which is negligible compared to the $\mathrm{NV}^{-}$center PL from the pillar (see Figure 6b). 
Keeping background PL from etched surfaces low is important as it limits the signal-tobackground ratio and consequently the achievable magnetic field sensitivity [32]. Figure 6c shows an exemplary optically detected magnetic resonance (ODMR) measured on single $\mathrm{NV}^{-}$centers in the structures. Here, we measured an ODMR contrast of $\sim 15 \%$. By investigating the coherence of the $\mathrm{NV}^{-}$centers in the nanostructures, we found a coherence time of $\mathrm{T}_{2} \leq 10 \mu \mathrm{s}$. We attributed this to the $\mathrm{NV}^{-}$centers' proximity to the surface which is in a good agreement with results from other groups measuring the coherence of shallow $\mathrm{NV}^{-}$centers in three-acid-cleaned SCD [33]. Considering the already low $T_{2}$, we cannot fully exclude a negative influence of the structuring on $T_{2}$.
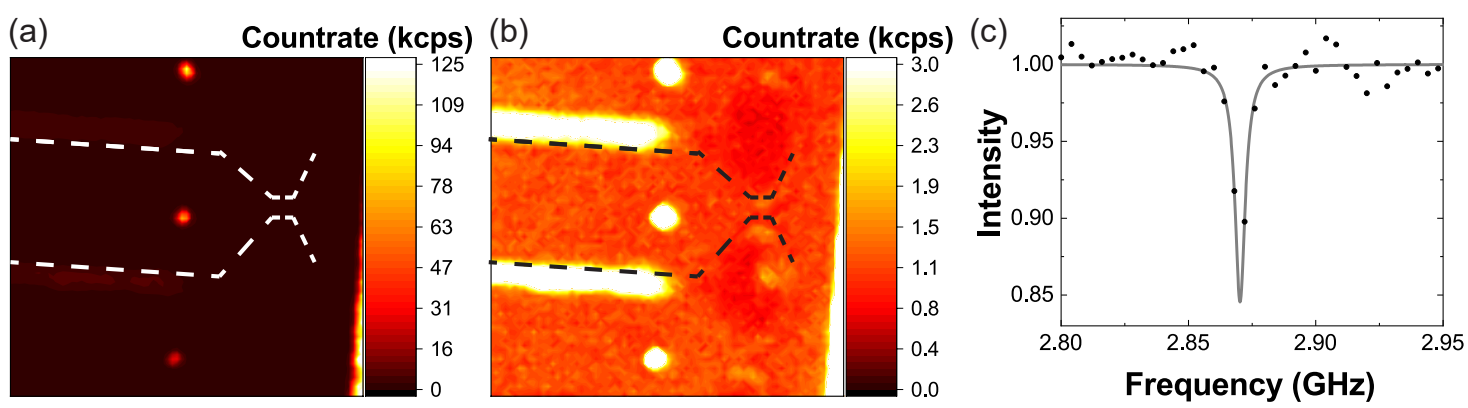

Figure 6. (a,b) PL map of SCD platforms with individual nanopillars fabricated with the process described in Section 4. The outer edge of the holding platform is indicated using a dashed line, while the pillars appear as bright spots. To enable comparing the background PL from the platforms with the PL of a few $\mathrm{NV}^{-}$centers in the pillars, we show the same PL map with two different scaling: individual $\mathrm{NV}^{-}$centers in the pillars show PL countrates above $125 \mathrm{kcps}$ at an excitation power of $500 \mu \mathrm{W}$ at $532 \mathrm{~nm}$ (a). (b) It is clearly discernible that the cantilevers show only a weak PL of $\sim 1 \mathrm{kcps}$. The enhanced background in-between the platform arises from a slight roughening between the structures. We detect $\mathrm{NV}^{-} \mathrm{PL}$ in the wavelength range $>650 \mathrm{~nm}$. (c) An exemplary optically detected magnetic resonance (ODMR) of one of the $\mathrm{NV}^{-}$centers in the pillar. The resonance (without an external magnetic field) at $2.87 \mathrm{GHz}$ is clearly visible and has a contrast of $\sim 15 \%$.

\section{Summary and Conclusions}

In this paper, we present a reliable technology for nanofabrication of SCD structures. We used our method to manufacture SCD scanning probes with shallowly embedded negative nitrogen vacancies. The method introduces an evaporated silicon adhesion layer on the SCD surface to ease adhesion and EBL with spin-coated HSQ-based Fox 16 resist. We present a methodology for the selective removal of our silicon adhesive/decharging layer with $\mathrm{SF}_{6}$ plasma. In areas protected by the HSQ mask, silicon layer survives etching as well as wet chemical removal of the residual HSQ mask and can be re-used for further nanofabrication, in our case for pillars on SCD platform. The shallowly implanted $\mathrm{NV}^{-}$centers survived the nanofabrication process. We found this method to be reliable, which is a considerable advancement in SCD nanofabrication technology that can be expanded to various kinds of SCD structures including SCD cantilever or cavity structures (e.g., photonic crystals).

Author Contributions: Funding acquisition, E.N.; Investigation, M.R., R.N., A.S. and E.N.; Methodology, M.R., R.N., A.S. and E.N; Supervision, E.N.; Writing—original draft, M.R., R.N. and E.N.; Writing—review and editing, M.R., R.N., A.S. and E.N.

Funding: This research was funded by NanoMatFutur grant of the German Ministry of Education and Research (FKZ13N13547).

Acknowledgments: We would like to acknowledge Sandra Wolff (TU Kaiserslautern, Germany) for help with electron beam evaporation, Jörg Schmauch (INM, Germany) for his help with acquiring high-quality SEM images and Rene Hensel (INM, Germany) for granting access to the ICP/RIE reactor. We acknowledge Michel Challier for his assistance and Andreas Ruh (Saarland University, Germany) for his help with EDX. We gratefully acknowledge cooperation and fruitful discussion with QNAMI (Basel), especially with Felipe Favaro De Oliveira and Patrick Maletinsky on the evaluation of our novel nanofab method. We note that the results presented in this study are filed for a patent, application number: EP19198772.6. 
Conflicts of Interest: The authors declare no conflict of interest.

\section{References}

1. Atatüre, M.; Englund, D.; Vamivakas, N.; Lee, S.Y.; Wrachtrup, J. Material platforms for spin-based photonic quantum technologies. Nat. Rev. Mater. 2018, 3, 38. [CrossRef]

2. Casola, F.; van der Sar, T.; Yacoby, A. Probing condensed matter physics with magnetometry based on nitrogen-vacancy centres in diamond. Nat. Rev. Mater. 2018, 3, 17088. [CrossRef]

3. Kucsko, G.; Maurer, P.; Yao, N.; Kubo, M.; Noh, H.; Lo, P.; Park, H.; Lukin, M. Nanometre-scale thermometry in a living cell. Nature 2013, 500,54-58. [CrossRef] [PubMed]

4. Teissier, J.; Barfuss, A.; Appel, P.; Neu, E.; Maletinsky, P. Strain coupling of a nitrogen-vacancy center spin to a diamond mechanical oscillator. Phys. Rev. Lett. 2014, 113, 020503. [CrossRef] [PubMed]

5. Dolde, F.; Doherty, M.W.; Michl, J.; Jakobi, I.; Naydenov, B.; Pezzagna, S.; Meijer, J.; Neumann, P.; Jelezko, F.; Manson, N.B.; et al. Nanoscale detection of a single fundamental charge in ambient conditions using the NV- center in diamond. Phys. Rev. Lett. 2014, 112, 097603. [CrossRef] [PubMed]

6. Maletinsky, P.; Hong, S.; Grinolds, M.; Hausmann, B.; Lukin, M.; Walsworth, R.; Loncar, M.; Yacoby, A. A robust scanning diamond sensor for nanoscale imaging with single nitrogen-vacancy centres. Nat. Nanotechnol. 2012, 7, 320-324. [CrossRef] [PubMed]

7. Neumann, P.; Kolesov, R.; Naydenov, B.; Beck, J.; Rempp, F.; Steiner, M.; Jacques, V.; Balasubramanian, G.; Markham, M.L.; Twitchen, D.J.; et al. Quantum register based on coupled electron spins in a room-temperature solid. Nat. Phys. 2010, 6, 249-253. [CrossRef]

8. Kurtsiefer, C.; Mayer, S.; Zarda, P.; Weinfurter, H. Stable solid-state source of single photons. Phys. Rev. Lett. 2000, 85, 290-293. [CrossRef]

9. Babinec, T.; Hausmann, B.; Khan, M.; Zhang, Y.; Maze, J.; Hemmer, P.; Loncar, M. A diamond nanowire single-photon source. Nat. Nanotechnol. 2010, 5, 195-199. [CrossRef]

10. Gruber, A.; Dräbenstedt, A.; Tietz, C.; Fleury, L.; Wrachtrup, J.; von Borczyskowski, C. Scanning confocal optical microscopy and magnetic resonance on single defect centers. Science 1997, 276, 2012-2014. [CrossRef]

11. Zaitsev, A. Optical Properties of Diamond: A Data Handbook; Springer: Berlin, Germany, 2001.

12. Hausmann, B.J.; Khan, M.; Zhang, Y.; Babinec, T.M.; Martinick, K.; McCutcheon, M.; Hemmer, P.R.; Loncar, M. Fabrication of diamond nanowires for quantum information processing applications. Diam. Relat. Mater. 2010, 19, 621-629. [CrossRef]

13. Grigorescu, A.; Hagen, C. Resists for sub-20-nm electron beam lithography with a focus on HSQ: State of the art. Nanotechnology 2009, 20, 292001. [CrossRef] [PubMed]

14. Neu, E.; Appel, P.; Ganzhorn, M.; Miguel-Sanchez, J.; Lesik, M.; Mille, V.; Jacques, V.; Tallaire, A.; Achard, J.; Maletinsky, P. Photonic nano-structures on (111)-oriented diamond. Appl. Phys. Lett. 2014, 104, 153108. [CrossRef]

15. Fuchs, P.; Challier, M.; Neu, E. Optimized single-crystal diamond scanning probes for high sensitivity magnetometry. New J. Phys. 2018, 20, 125001. [CrossRef]

16. Appel, P.; Neu, E.; Ganzhorn, M.; Barfuss, A.; Batzer, M.; Gratz, M.; Tschöpe, A.; Maletinsky, P. Fabrication of all diamond scanning probes for nanoscale magnetometry. Rev. Sci. Instrum. 2016, 87, 063703. [CrossRef]

17. Lillie, S.E.; Broadway, D.A.; Dontschuk, N.; Zavabeti, A.; Simpson, D.A.; Teraji, T.; Daeneke, T.; Hollenberg, L.C.; Tetienne, J.P. Magnetic noise from ultrathin abrasively deposited materials on diamond. Phys. Rev. Mater. 2018, 2, 116002. [CrossRef]

18. Xie, L.; Zhou, T.X.; Stöhr, R.J.; Yacoby, A. Crystallographic orientation dependent reactive ion etching in single crystal diamond. Adv. Mater. 2018, 30, 1705501. [CrossRef]

19. Volpe, P.N.; Muret, P.; Omnes, F.; Achard, J.; Silva, F.; Brinza, O.; Gicquel, A. Defect analysis and excitons diffusion in undoped homoepitaxial diamond films after polishing and oxygen plasma etching. Diam. Relat. Mater. 2009, 18, 1205-1210. [CrossRef]

20. Naamoun, M.; Tallaire, A.; Silva, F.; Achard, J.; Doppelt, P.; Gicquel, A. Etch-pit formation mechanism induced on HPHT and CVD diamond single crystals by $\mathrm{H}_{2} / \mathrm{O}_{2}$ plasma etching treatment. Phys. Stat. Solidi 2012, 209, 1715-1720. [CrossRef] 
21. Challier, M.; Sonusen, S.; Barfuss, A.; Rohner, D.; Riedel, D.; Koelbl, J.; Ganzhorn, M.; Appel, P.; Maletinsky, P.; Neu, E. Advanced fabrication of single-crystal diamond membranes for quantum technologies. Micromachines 2018, 9, 148. [CrossRef]

22. Radtke, M.; Nelz, R.; Render, L.; Neu, E. Plasma treatments and photonic nanostructures for shallow nitrogen vacancy centers in diamond. arXiv 2019, submitted.

23. De Oliveira, F.F.; Momenzadeh, S.A.; Wang, Y.; Konuma, M.; Markham, M.; Edmonds, A.M.; Denisenko, A.; Wrachtrup, J. Effect of low-damage inductively coupled plasma on shallow nitrogen-vacancy centers in diamond. Appl. Phys. Lett. 2015, 107, 073107. [CrossRef]

24. Kato, Y.; Kawashima, H.; Makino, T.; Ogura, M.; Traoré, A.; Ozawa, N.; Yamasaki, S. Estimation of inductively coupled plasma etching damage of boron-doped diamond using X-Ray photoelectron spectroscopy. Phys. Status Solidi (A) 2017, 214, 1700233. [CrossRef]

25. Krueger, A.; Lang, D. Functionality is key: Recent progress in the surface modification of nanodiamond. Adv. Funct. Mater. 2012, 22, 890-906. [CrossRef]

26. Challier, M.; Nelz, R.; Kiendl, B.; Slablab, A.; Radtke, M.; Mueller, F.; Krueger, A.; Neu, E. Wet Chemical Fluorine Treatment of Diamond: Towards Hydrophobic Diamond Nano-Devices for Single NV Sensing. Neu, E. Saarland University, Saarbrücken, Germany. Unpublished work, 2019.

27. dAgostino, R.; Flamm, D.L. Plasma etching of $\mathrm{Si}$ and $\mathrm{SiO}_{2}$ in $\mathrm{SF}_{6}$ mixtures. J. Appl. Phys. 1981, 52, $162-167$. [CrossRef]

28. Morshed, M.; Daniels, S. Electron density and optical emission measurements of $\mathrm{SF}_{6} / \mathrm{O}_{2}$ plasmas for silicon etch processes. Plasma Sci. Technol. 2012, 14, 316-320. [CrossRef]

29. Nelz, R.; Fuchs, P.; Opaluch, O.; Sonusen, S.; Savenko, N.; Podgursky, V.; Neu, E. Color center fluorescence and spin manipulation in single crystal, pyramidal diamond tips. Appl. Phys. Lett. 2016, 109, 193105. [CrossRef]

30. Nelz, R.; Görlitz, J.; Herrmann, D.; Slablab, A.; Challier, M.; Radtke, M.; Fischer, M.; Gsell, S.; Schreck, M.; Becher, C.; et al. Toward wafer-scale diamond nano-and quantum technologies. Apl. Mater. 2019, 7, 011108. [CrossRef]

31. Nelz, R.; Radtke, M.; Slablab, A.; Kianinia, M.; Li, C.; Xu, Z.Q.; Bradac, C.; Aharonovich, I.; Neu, E. Near-field energy transfer between a luminescent 2D material and color centers in diamond. arXiv 2019, 1907, 12248.

32. Rondin, L.; Tetienne, J.P.; Hingant, T.; Roch, J.F.; Maletinsky, P.; Jacques, V. Magnetometry with nitrogen-vacancy defects in diamond. Rep. Prog. Phys. 2014, 77, 056503. [CrossRef] [PubMed]

33. Sangtawesin, S.; Dwyer, B.L.; Srinivasan, S.; Allred, J.J.; Rodgers, L.V.; De Greve, K.; Stacey, A.; Dontschuk, N.; O'Donnell, K.M.; Hu, D.; et al. Origins of diamond surface noise probed by correlating single spin measurements with surface spectroscopy. Phys. Rev. 2019, 9, 031052. [CrossRef] 